\title{
Ansiedade infantil antes e após o tratamento endodôntico
}

\author{
Anxiety child before and after endodontical treatment
}

\author{
Daiani Jovanowichs Ramos* \\ Jéssica Carolina Larini Oliveira** \\ Marília Leão Goettmes ${ }^{* * *}$ \\ Luiza Helena Almeida****
}

\section{Resumo}

Objetivo: avaliar o grau de ansiedade das crianças antes e após o tratamento endodôntico, por meio do teste Venham Picture Teste (VPT) modificado, e a percepção dos responsáveis diante do atendimento dos filhos. Sujeitos e método: trata-se de um estudo observacional, do tipo transversal, em que foram selecionadas 50 crianças entre 4 e 8 anos de idade, atendidas nas disciplinas de estágios supervisionados em Odontopediatria e Ortodontia I e II da Faculdade Avantis, Santa Catarina. As crianças que participaram da pesquisa foram distribuídas em dois grupos: as que passaram por consultas com terapia endodôntica (Grupo $A=25$ crianças); e as que passaram por consultas menos invasivas (Grupo $B=25$ crianças). O teste VPT foi aplicado em dois momentos: antes e após o atendimento odontológico. Os responsáveis legais pelas crianças também responderam perguntas relacionadas à ansiedade. Resultados: a maioria das crianças apresentou nível baixo de ansiedade antes e após os procedimentos, $84 \%$ e $92 \%$ para os grupos $A$ e $B$, respectivamente. A respeito da ansiedade dos responsáveis, a maioria encontrava-se relaxada durante o procedimento do filho. Conclusão: a maioria das crianças apresentou um nível baixo de ansiedade em ambos os grupos e os responsáveis estavam se sentindo relaxados durante o atendimento dos filhos.

Palavras-chave: Ansiedade. Atendimento odontológico. Crianças. Endodontia. Odontopediatria.

\section{Introdução}

A dentição decídua exerce influência significativa no crescimento e desenvolvimento da criança. Essa influência ocorre tanto sistemicamente, na respiração, fonação, alimentação, quanto localmente, promovendo a manutenção de espaço para a dentição permanente e estimulando o crescimento da maxila ${ }^{1,2}$. Nos casos em que os dentes decíduos evoluem para inflamação pulpar irreversível ou necrose, sempre que possível, para sua manutenção no arco, indica-se o tratamento endodôntico ${ }^{3}$, que compreende a limpeza e a modelagem dos condutos radiculares seguidas pela obturaçã $0^{4}$. Esse tratamento, por ser mais demorado, pode trazer algum incômodo ao paciente infantil, para tanto, o profissional precisa ter conhecimento dos diferentes estágios do desenvolvimento psicológico da criança e de seu comportamento ${ }^{5}$.

O comportamento infantil é definido por experiências, vivências e influências que a criança recebe do meio em que vive. A falta de tratamento, a presença de dor no tratamento anterior e a forma com que os pais lidam com a ansiedade e como transmitem essa informação à criança são fatores decisivos para as atitudes dentro do consultório odontológico ${ }^{6}$. No estudo de Goes et al. ${ }^{7}$ (2009), a presença de história odontológica relevante, ou seja, primeira vez ao dentista ou experiência negativa em consultas anteriores, representou chances muito maiores de apresentar ansiedade infantil no tratamento odontológico. 
O teste Venham Picture Test (VPT) foi preconizado para medir a ansiedade infantil durante o tratamento odontológico e é um instrumento no qual se usa um conjunto de figuras, entre as quais a criança que está sendo pesquisada escolhe a que mais se identifica no momento. Apresentam-se às crianças oito pares de figuras com uma menina ou um menino, conforme o sexo do participante, que expressam várias reações; diante delas, as crianças são estimuladas a escolher as figuras que mais refletem suas emoções. Assim sendo, o teste VPT modificado, validado no Brasil, pode auxiliar na avaliação do estado emocional da criança durante o procedimento . $^{\text {. }}$

Sabe-se que o vínculo e a confiança entre o profissional cirurgião-dentista e o paciente infantil são influenciados por adequado manejo psicológico da criança $^{9}$. Sendo assim, o controle da ansiedade infantil se torna indispensável para o atendimento no consultório odontológico. Tendo em vista que ainda há necessidade de realizar tratamento endodôntico em crianças, é necessário que o odontopediatra saiba prevenir e avaliar a ansiedade. Assim, o objetivo do presente trabalho foi avaliar o grau de ansiedade de crianças antes e após o tratamento endodôntico, utilizando o teste VPT modificado, e a percepção dos responsáveis perante o atendimento dos filhos. A hipótese do artigo era a de que crianças que passariam por tratamento endodôntico apresentariam mais ansiedade quando comparadas às crianças que receberiam tratamentos menos invasivos.

\section{Sujeitos e método}

Este estudo foi previamente encaminhado ao Comitê de Ética em Pesquisa da Faculdade Avantis, tendo sido aprovado sob o parecer $\mathrm{n}^{\mathrm{o}}$ 1.953.879, atendendo aos requisitos da Resolução no ${ }^{\circ}$ 466/1996, do Conselho Nacional de Saúde. O desenho do estudo foi observacional, do tipo transversal. Foram incluídas na pesquisa 50 crianças entre 4 e 8 anos, as quais foram atendidas nas disciplinas de estágios supervisionados em Odontopediatria e Ortodontia I e II do Curso de Odontologia da Faculdade Avantis, SC, durante o período de março de 2016 a setembro de 2017.
A amostra foi selecionada por conveniência. As crianças foram divididas em dois grupos: grupo A, com crianças que receberam tratamento endodôntico; e grupo B, com crianças que receberam tratamentos menos invasivos: como profilaxia, adequação do meio e fluorterapia. A partir dos prontuários dos pacientes, foram coletados dados referentes a sexo, idade, nível escolar, renda familiar, estrutura familiar, número de consultas odontológicas, tipo de controle da dor realizado (com anestesia, sem anestesia) e tipo de manejo utilizado (com contenção protetora ou sem contenção protetora).

As crianças foram escolhidas aleatoriamente, as do grupo A deveriam ter pelo menos uma indicação de tratamento endodôntico, e as do grupo B, ter recebido atendimento de rotina, com procedimentos menos invasivos, que não geraram desconforto ou ansiedade.

Todas as consultas foram conduzidas no mesmo ambiente. Os pesquisadores dirigiram-se até as crianças e seus responsáveis que aguardavam para o início do atendimento odontológico na clínica de pacientes infantis da Faculdade Avantis, explicando os objetivos da pesquisa e perguntando se concordavam em participar, após a assinatura do termo de consentimento livre e esclarecido, iniciaram-se os testes e os questionários.

Para a avaliação da ansiedade da criança, foi utilizada a escala do teste VPT, modificada por Ramos-Jorge et al. ${ }^{8}$ (2006) (Figura 1). As crianças, já no ambiente odontológico, ou seja, na cadeira, foram apresentadas à escala VPT modificada e escolheram as figuras que apresentavam a etnia e $o$ sexo correspondentes. Após a escolha, era indagado, de maneira clara, pelo examinador previamente calibrado, o seguinte: "Eu gostaria que você apontasse para o(a) menino(a) que está sentindo o mesmo que você está sentindo agora. Olhe cuidadosamente para os rostos nas figuras e veja como eles se sentem". Cada um dos oito pares de figuras era mostrado separadamente para a criança. Para a figura que revelava um sentimento negativo era atribuído um ponto na avaliação. A soma da avaliação de todos os pares de figuras pode variar de zero a oito, sendo que zero representa crianças livres de ansiedade, um a três - baixo nível de ansiedade, quatro a seis - nível médio de ansiedade e oito a nove - alto nível de ansiedade ${ }^{8}$. A escala VPT foi aplicada previamente e logo após o atendimento odontológico. 
Código de Identificação da Criança:
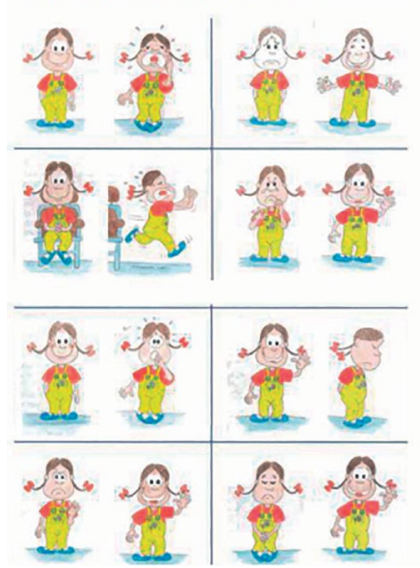

Figura 1 - Escala Venham Picture Test modificada

Fonte: elaboração dos autores.

Os responsáveis legais foram questionados sobre a sua percepção diante do tratamento odontológico do filho. A pergunta era: Como o(a) senhor(a) se sente quando seu(sua) filho(a) está sentado(a) na cadeira do dentista? $\mathrm{E}$ as possíveis respostas eram: relaxada(o); desconfortável; ansiosa(o); e tão ansiosa(o), que começo a suar e me sentir mal.

Em seguida, os dados foram digitados em um banco de dados do programa Microsoft Excel e analisados no programa Stata 10.0. Os dados foram submetidos à análise estatística descritiva e apresentados em forma de frequências relativas e seus percentuais. Para a comparação dos grupos, foi aplicado o teste exato de Fisher.

\section{Resultados}

A escala VPT foi aplicada em 50 crianças com idades entre 4 e 8 anos, sendo que 25 fizeram parte do grupo A e 25 do grupo B. A Tabela 1 mostra as características da amostra estudada. A Tabela 2 mostra os dados obtidos no grupo A e no grupo B, de acordo com o nível de ansiedade para cada criança.

Tabela 1 - Frequências absolutas e relativas da amostra estudada $(n=50)$

\begin{tabular}{l|c}
\hline Renda familiar & $\mathrm{n}(\%)$ \\
\hline Até um salário mínimo & $20(40 \%)$ \\
Dois a três salários mínimos & $24(48 \%)$ \\
Acima de três salários mínimos & $6(12 \%)$ \\
\hline Estrutura familiar & $32(64 \%)$ \\
\hline Mora com pai e mãe & $13(26 \%)$ \\
Apenas com a mãe & $5(10 \%)$ \\
Outras situações (avós e tios) & $19(38 \%)$ \\
\hline Idade & $18(36 \%)$ \\
\hline 4-5 anos & $13(26 \%)$ \\
6-7 anos & \\
8 anos & $10(20 \%)$ \\
\hline Técnica de manejo & $40(80 \%)$ \\
\hline Com contenção
\end{tabular}

Fonte: elaboração dos autores.

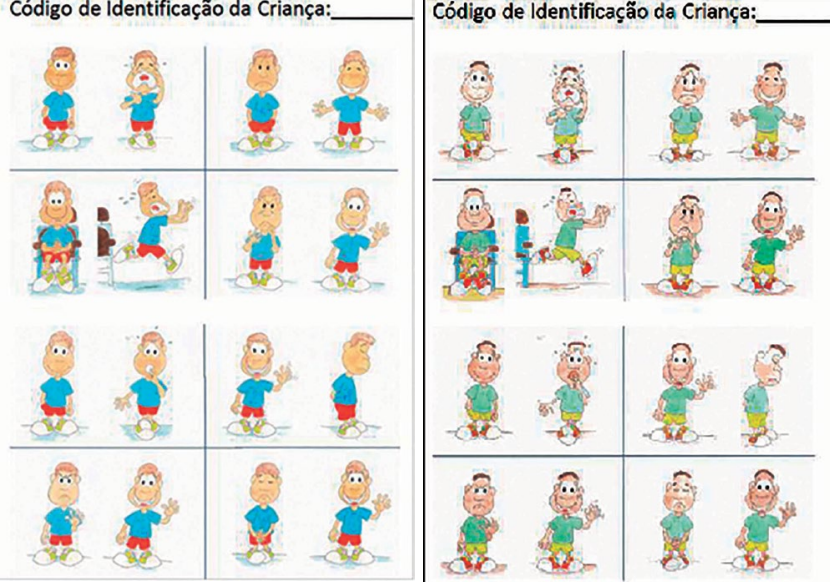

Tabela 2 - Distribuição dos grupos A e B segundo a escala VPT (50 crianças)

\begin{tabular}{c|c|c|c}
\hline $\begin{array}{c}\text { Nível de } \\
\text { ansiedade }\end{array}$ & $\begin{array}{c}\text { Antes do } \\
\text { procedimento }\end{array}$ & $\begin{array}{c}\text { Após o } \\
\text { procedimento }\end{array}$ & $\mathrm{P}<0,05$ \\
\hline Grupo A & 21 & 0,99 \\
\hline Baixo & 21 & 2 & \\
Médio & 4 & 2 & \\
Alto & 0 & 23 & 0,23 \\
\hline Grupo B & \multicolumn{3}{|l}{} \\
\hline Baixo & 23 & 2 & \\
Médio & 0 & 0 & \\
Alto & 2 & 0,083 & \\
\hline $\mathrm{P}<0,05$ & 0,670 & & \\
\hline
\end{tabular}

Fonte: elaboração dos autores.

Nota: Diferenças estatísticas entre os grupos na linha e diferença estatística na coluna dentro do mesmo grupo.

Segundo o teste, $84 \%$ (21) das crianças do grupo A apresentaram nível baixo de ansiedade antes e após o procedimento; $16 \%$ (4), nível médio de ansiedade antes do procedimento, índice que, ao final, reduziu para $8 \%$ (2). O nível alto de ansiedade foi observado em apenas 2 crianças (8\%) ao final de procedimentos, as quais se encontravam no nível médio de ansiedade antes do atendimento. Não houve diferença estatisticamente significante antes e após os procedimentos para o grupo A.

No grupo B, predominou o nível baixo de ansiedade, em $92 \%$ (23), tanto antes quanto após o procedimento. No nível médio de ansiedade, enquadraram-se $8 \%$ (2) das crianças somente após o procedimento; $2(8 \%)$ crianças tiveram nível alto apenas antes do procedimento. Não houve diferença estatisticamente significante antes e após os procedimentos para o grupo B, e nem entre os grupos A e B.

Os resultados obtidos por meio da pesquisa com os responsáveis legais mostraram que $60 \%$ encontravam-se relaxados durante o procedimento do filho, 30\% encontravam-se ansiosos, 6\% desconfortáveis e $4 \%$ sentiam-se muito ansiosos (Tabela 3 ). 
Tabela 3 - Distribuição dos resultados obtidos após a entrevista direcionada aos responsáveis

Como se sentem durante o procedimento

\section{Relaxada(o)}

Ansiosa(o)

Desconfortável

Tão ansiosa(o) que começa (o) a suar e se sentir mal Fonte: elaboração dos autores.

Na Figura 2, pode-se observar a predominância de crianças e responsáveis com nível baixo de ansiedade.

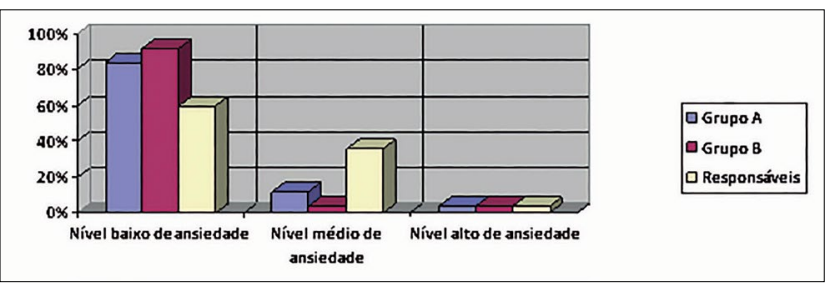

Figura 2 - Nível de ansiedade dos grupos estudados e seus responsáveis

Fonte: elaboração dos autores.

\section{Discussão}

O maior desafio para o atendimento odontopediátrico é o manejo do comportamento dos pacientes. Estudos relacionados a essa temática são de extrema importância para nortear o cirurgião-dentista acerca de metodologias adequadas para o atendimento. Medo e ansiedade estão interligados, mas seus conceitos são distintos. $\mathrm{O}$ medo faz parte do desenvolvimento da infância normal da criança, pode ser transitório ou perdurar por longos períodos, como o medo do atendimento odontológico. A ansiedade é tida como resposta diante de uma situação de ameaça, não é bem definida nem objetivamente presente ${ }^{7}$.

Neste estudo, a ansiedade das crianças, assim como a de seus responsáveis, durante atendimentos odontológicos, avaliada por meio da escala VPT, manteve-se em um baixo nível, apesar de não haver diferença estatisticamente significante, sendo assim, a hipótese do artigo foi rejeitada.

Segundo Albuquerque et al..$^{9}(2010)$, mesmo que o dentista possua habilidades de lidar com crianças, para que haja uma melhora significativa na relação entre o odontopediatra e o paciente, são necessários conhecimentos sobre psicologia e técnicas de manejo do comportamento infantil. Quando existe segurança por parte do dentista em relação às suas habilidades de manejo de conduta, há uma maior cooperação do paciente infantil. Neste trabalho, $84 \%$ das crianças do grupo A e $92 \%$ do grupo B foram classificadas, segundo o teste VPT modificado, com baixo nível de ansiedade. Os resultados apontaram que as crianças atendidas na instituição, de maneira geral, apresentam-se tranquilas nas consultas odontológicas, independentemente do tratamento a ser executado. Além disso, contatou-se que os aca- dêmicos que realizaram os procedimentos estavam preparados para realizar o tratamento, invasivo ou não, usando a técnica de manejo correta naquele momento.

Resultados similares foram encontrados no estudo de Oliveira et al. ${ }^{6}$ (2012), no qual os autores verificaram que, a maioria das crianças entrevistadas apresentaram-se livres ou com baixo nível de ansiedade. Essa mensuração demonstra que a ansiedade da criança durante o atendimento odontológico depende não só do preparo prévio dos pais, mas também da habilidade do cirurgião-dentista e de sua equipe em manejá-la ${ }^{9,10}$.

No nível médio de ansiedade, enquadraram-se quatro crianças do grupo $\mathrm{A}$, antes do procedimento. Este resultado pode ser explicado pelo fato de a criança não conhecer o que seria executado, apenas reflexões das experiências prévias vividas ou até mesmo daquilo que lhe foi relatado. Tal situação pode ser definida pelo medo, que é uma inquietação emocional diante de uma ameaça, interligada com a ansiedade, que nos mantém em alerta, em estado de opressão, com medo difuso, acompanhado de sinais somáticos, palpitações e suores ${ }^{11}$.

Segundo Ramos-Jorge et al. ${ }^{12}$ (2011), o medo de dentista pode ser considerado algum temor das primeiras experiências, tratamentos odontológicos malsucedidos e/ou situações que geraram algum desconforto físico ou psicológico; ou seja, mesmo não sendo um procedimento tão invasivo, essas circunstâncias podem ter sido reflexo dos achados encontrados.

Da mesma forma, as crianças do grupo B, com alto índice de ansiedade, após o procedimento, estavam com baixo nível de ansiedade. Sabe-se que crianças têm um receio inicial prévio à consulta odontológica, contudo, após ser superado, cria-se um vínculo entre paciente e dentista, de modo que a criança passa a se sentir mais tranquila durante a consulta $^{6}$. A relação interpessoal entre o cirurgião-dentista e o paciente torna a criança mais confiante, aumenta a possibilidade da tolerância da criança ao procedimento odontológico, podendo ocorrer uma maior facilidade de o cirurgião-dentista convencer a criança das frequências das consultas ${ }^{11}$.

Nos tratamentos odontológicos mais longos, em que há a necessidade do uso de anestesia, como é o caso da terapia endodôntica, a criança poderá, algumas vezes, apresentar cansaço durante a execução do procedimento e passar a desenvolver ansiedade prévia à consulta ou ao final ${ }^{6}$. Pôde-se observar que, entre as crianças do grupo A que receberam anestesia, apenas $2(8 \%)$ apresentaram nível alto de ansiedade, resultados semelhantes foram encontrados no grupo B, no qual não houve o uso de anestesia. Esperava-se que houvesse diferenças entre os grupos, no entanto, isso não ocorreu. Similar resultado foi observado em crianças expostas a imagens positivas e imagens neutras referentes ao tratamento odontológico, e não houve diferença significativa ${ }^{13,14}$. 
A amostra total apresentou heterogeneidade quanto à idade, variando de 4 a 8 anos, e apresentou alto índice de crianças com baixo nível de ansiedade antes e após o procedimento (88\%), o que reforça o preparo dos acadêmicos quanto ao uso adequado das técnicas de manejo do comportamento infantil, para diferentes faixas etárias.

O comportamento da criança perante o tratamento odontológico depende, em grande parte, da sua maturidade psicológica. Â medida que o processo de amadurecimento acontece, a criança passa, comumente, a aceitar melhor o tratamento ${ }^{5}$. A relação harmoniosa entre os acadêmicos de Odontologia e os pacientes e os responsáveis é outro fator que estabelece um atendimento humanístico durante as aulas práticas de odontopediatria ${ }^{15}$.

A ansiedade de pais e mães bem como a maneira como esses reagem às impressões dos filhos e à preparação da criança para 0 atendimento odontológico têm muita influência no seu comportamento ${ }^{16-18}$. Uma criança que teve uma boa preparação pelos pais tem seu medo diminuído e, consequentemente, também a sua resistência em relação ao tratamento proposto ${ }^{19-21}$. Nos resultados encontrados nesta pesquisa, $60 \%$ dos responsáveis pelas crianças sentiam-se relaxados durante o procedimento, $30 \%$ ansiosos, $6 \%$ desconfortáveis e $4 \%$ altamente ansiosos. Foi observado um maior número de responsáveis ansiosos no grupo A, se comparado ao grupo B.

O estudo apresenta algumas limitações. A amostra foi por conveniência e, portanto, os resultados não podem ser extrapolados para toda a população infantil do município. Além disso, os procedimentos odontológicos foram realizados por diferentes alunos e, possivelmente, pode ter ocorrido influência nos resultados devido a diferenças de personalidade e formas de abordagem. Por se tratar de uma escola, os pacientes são atendidos em um mesmo ambiente, e o comportamento de uma criança eventualmente pouco colaboradora pode ter influenciado na resposta de ansiedade dos participantes.

Foi importante ter um panorama sobre a ansiedade das crianças atendidas na clínica infantil para avaliar o manejo do comportamento realizado pelos acadêmicos bem como para demostrar que, para esta amostra, o tratamento endodôntico, muitas vezes temorizado por familiares, pôde ser realizado com crianças sem trazer consequências comportamentais, como elevar o nível de ansiedade, por exemplo.

\section{Conclusão}

Apesar de não ter sido verificada diferença estatística significativa entre os grupos avaliados, os resultados observados demostram que, na amostra, a maior parte das crianças relatou baixos níveis de ansiedade antes e após o tratamento endodôntico e os procedimentos menos invasivos. Os responsáveis legais também estavam se sentindo relaxados durante $o$ atendimento de seus filhos.

\section{Abstract}

Objective: evaluate the degree of anxiety in children before and after endodontic treatment, through the Venham Picture Test (VPT), and the perception of those responsible for the care of the children. Subjects e method: this is an observational, cross-sectional study, in which 50 children between 4 and 8 years of age attended the supervised stages in Pediatric Dentistry and Orthodontics I and II of the Faculdade Avantis, SC. The children who were interviewed with the VPT test to measure the degree of anxiety were divided into 2 groups: children who received consultations with endodontic therapy (Group $A=25$ children); and less invasive consultations (Group $B=25$ children). The VPT test was applied in two moments: before and after dental care. Legal officials also answered questions related to anxiety. Results: the majority of the children presented low level of anxiety before and after the procedures, $84 \%$ and $92 \%$ for groups $A$ and $B$ respectively. Regarding maternal anxiety, most were relaxed during the child's procedure. Conclusion: most of the children presented a low level of anxiety in both groups. Parents were feeling relaxed during the care of their children.

Keywords: Anxiety. Dental care. Child. Endodontics. Fear. Pediatric Dentistry.

\section{Referências}

1. Brothwell DJ. Guidelines on the use of space maintainers following premature loss of primary teeth. J Can Dent Assoc 1997; 63(5):753-66.

2. Da Costa CC, Almeida CI. Clinical comparative study of the effects of two types of mandibular space-regaining devices. Gen Dent 2006; 51(3):120-6.

3. Fuks AB. Vital pulp therapy with new materials for primary teeth: new directions and treatment perspectives. Pediatr Dent 2008; 30(3):211-9.

4. Ørstavik D. Materials used for root canal obturation: technical, biological and clinical testing. Endodontic Topics 2005; 12(1):25-38.

5. Ramos-Jorge ML, Paiva SM. Comportamento infantil no ambiente odontológico: aspectos psicológicos e sociais. J Bras Odontopediatr Odontol Bebê 2003; 6(29):70-4.

6. Oliveira MF, Morais MVM, Cardoso DD. Avaliação da ansiedade infantil prévio ao tratamento odontológico. Pool UEPG Cienc Biol 2012; 18(1):31-7.

7. Goes MPS, Domingues MC, Couto GBL, Barreira AK. Ansiedade, medo e sinais vitais dos pacientes infantis. Revista Odonto 2009; 9(1):39-44.

8. Ramos-Jorge ML, Marques LS, Pavia SM, Serra-negra JM, Pordeus IA. Predictive factors for child behavior in the dental environment. Eur Arch Paediatr Dent Leeds 2006; 7(4):252-6.

9. Albuquerque CM, Gouvêa CVP, Moraes RCM, Barros RN, Couto CF. Principais técnicas de controle de comportamento em Odontopediatria. Arq Odontologia 2010; 46(02):110-5.

10. Singh KA, Moraes ABA, Ambrosano GMB. Medo, ansiedade e controle relacionados ao tratamento odontológico. Pesqui Odontol Bras 2000; 14(2):131-6.

11. Nagano HCM. Dilemas e reflexões de odontopediatras sobre estratégias de manejo do comportamento infantil [Tese de Doutorado]. Florianópolis: Faculdade de Odontologia; Universidade Federal de Santa Catarina; 2010. 
12. Ramos-Jorge ML, Ramos-Jorge J, Andrade RGV, Marques LS. Impact of exposure to positive images on dental anxiety among children: a controlled trial. Eur Arch Paediatr Dent 2011; 12(4):195-9.

13. Pereira VZ, Barreto RC, Pereira GAS, Cavalcanti HRBB. Evaluation of the level of anxiety in patients undergoing dental treatment. Rev Bras Ciências da Saúde 2013; 17(1):55-64.

14. Hass MG, Jardim COL, Sousa AM. Influência da vestimenta do cirurgião-dentista e do ambiente do consultório odontológico na ansiedade de crianças pré-escolares durante consulta odontológica: resultados de um estudo piloto. RFO UPF 2016; 21(2):201-7.

15. Goettems ML, Ardengui TM, Romano AR, Demarco FF, Torriani DD. Influence of maternal dental anxiety on the child's dental caries experience. Caries Res 2012; 46(1):3-8.

16. Karibe H, Aoyagi-Naka K, Koda A. Maternal anxiety and child fear during dental procedures: a preliminary study. J Dent Child (Chic) 2014; 81(2):72-7.

17. Majstorovic M, Morse DE, Do D, Lim LL, Herman NG, Moursi AM. Indicators of dental anxiety in children just prior to treatment. J Clin Pediatr Dent 2014; 39(1): 12-7.

18. Barbosa CSA, Toledo OAD. Uso de técnicas aversivas de controle de comportamento em odontopediatria. J Bras Odontopediatr Odontol Bebê 2003; 6(29):76-82.

19. Meira-Filho MMO, Araújo DTC, Menezes VA, Garcia AFG. Atendimento odontológico da criança: percepção materna. RGO 2009; 57(3):311-5.

20. Oliveira MF, Moraes MVM, Evarista PCS. Avaliação da ansiedade dos pais e crianças frente ao tratamento odontológico. Pesqu Bras Odontopediatria Clínica Integrada 2012; 12(4):483-9

21. Araújo SM, Silveira EG, Mello LD, Caregnato M, Da'1 Costa GV. Ponto de vista dos pais em relação a sua presença durante $\mathrm{o}$ atendimento odontológico de seus filhos. Salusvita 2010; 29(2):17-27.

\section{Endereço para correspondência:}

Luiza Helena Almeida

Centro de Saúde - Odontologia

Av. Marginal Leste, 3600 - Estados

CEP 88339-125 - Balneário Camboriú, SC, Brasil

Telefone: (47) 992649992

E-mail: luizahelenadentista@hotmail.com

Recebido: 05/10/17. Aceito: 06/12/17. 